\title{
Funky-design-spaces: Interactive Environments for Creativity Inspired by Observing Designers Making Mood Boards
}

\author{
Andrés Lucero \\ Mads Clausen Institute, University of Southern Denmark, Kolding, Denmark \\ lucero@acm.org
}

\begin{abstract}
Research in surface computing has traditionally been driven by technology. A project that explored ways to provide support for professional users in their work with novel technology by focusing on people instead is presented. A co-design approach was applied by systematically involving end users (i.e., industrial designers) throughout the design process. In a series of activities with fifty designers, the creation of mood boards was identified as an important task for them, and studied in depth. The funky-design spaces vision of a holistic design studio housing interconnected tools that support the creation of mood boards was co-designed, brought to life in two prototypes, and evaluated with designers. The results suggest these environments could stimulate designers to break away from their desks and encourage collaboration with more people.
\end{abstract}

Keywords. Design Practice · Creativity · Co-Design · Tabletops · Displays.

\section{Introduction}

The field of human-computer interaction (HCI) has been investigating how people interact with computer systems at work, at home, and more recently in open public spaces, in an attempt to help people achieve their goals. Within HCI, researchers have already identified the potential behind interactive horizontal (e.g., DigitalDesk [27], ReacTable [11]) and vertical surfaces (e.g., DynaWall [23], ambient displays [25]) as a more natural and familiar setting to design collaborative interactions. Research in this area has traditionally been driven by technology.

A research project that explored ways in which novel technologies can provide support for professional users (i.e., industrial designers) in their work is presented. A co-design approach was applied by systematically involving fifty practicing designers throughout the design process in a series of activities. First, cultural probes [8] were used to understand design practice and identify an important task for designers (i.e., mood-board making). Second, retrospective interviews based on contextual inquiry [10] were carried out in the Netherlands and Finland to understand what the creation of mood boards entails. Third, designers were invited to co-design workshops (i.e., dialogue-labs [16]) in both countries to collaboratively create novel tools that support the creation of mood boards. Finally, designers were invited to prototype evaluations 
of two resulting tools from the co-design sessions (i.e., the Funky Coffee Table [14] and Funky Wall [15]). The structure of the paper is as follows. First, two studies on design practice and mood board creation are presented. Next, the results of co-design workshops are introduced, which lead to the funky-design-spaces. Then, the two resulting tools and the outcome of a joint evaluation are presented. Finally, limitations of this work and future improvements are discussed, followed by conclusions.

\section{Design Practice}

With the aim to get a broad understanding of design practice and identify an important activity that could be supported by novel technologies, a first study with industrial designers was conducted. The study took place in design studios and looked into what designers do there, their activities, the places and objects they use in the practice of industrial design. There are many studies of design practice, focusing on different design disciplines and specific stages of the design process. These include studies with knitwear designers to facilitate communication within design teams [6], and of how product designers keep their informal collections of visual material [12].

\subsection{Method, Participants and Procedure}

The method used was design probes [8]. Regarding its main characteristics, probes: 1) are based on user participation by means of self-documentation, 2) look at the user's personal context and perceptions, and 3) have an exploratory character [18]. One main advantage of applying probes includes collecting participant data over an extended period of time (e.g., one week), which in turn allows people to reflect on what they are being asked and the answers they have provided on the previous days.

The probes study was conducted in the Netherlands with 10 practicing industrial designers. All participants had at least two years of design practice experience (nine years on average), with varying educational level (university or academy), age (between 24 and 50), and gender (6 male, 4 female). A wide variety of work contexts was obtained, ranging from an office in a large company, to freelance work performed at home, and sometimes combined with part-time teaching at a university.

Participants worked on the probes in their design studio and home for a period of one week (i.e., seven consecutive days) and freely chose the starting day. The probe kit contained a design-studio diary including: 1) a timeline to probe the daily thoughts and activities of the participants, 2) closed questions covering different aspects of routines, collaboration, and use of technology, 3) open questions to make people tell stories and express their opinions, 4) a map to allow for self-expression, and 5) a drawing exercise (i.e., ideal design studio) to probe the dreams and aspirations of the participants. The kit also included a single-use camera (i.e., 36 exposures) to illustrate interesting activities, places and objects, and a 'Picture Record Table' to keep track of their pictures. Two researchers processed the data from the diaries and cameras (i.e., 200 photos) for interpretation. General findings were formulated and presented back to the participants during a workshop to triangulate the interpretation. 

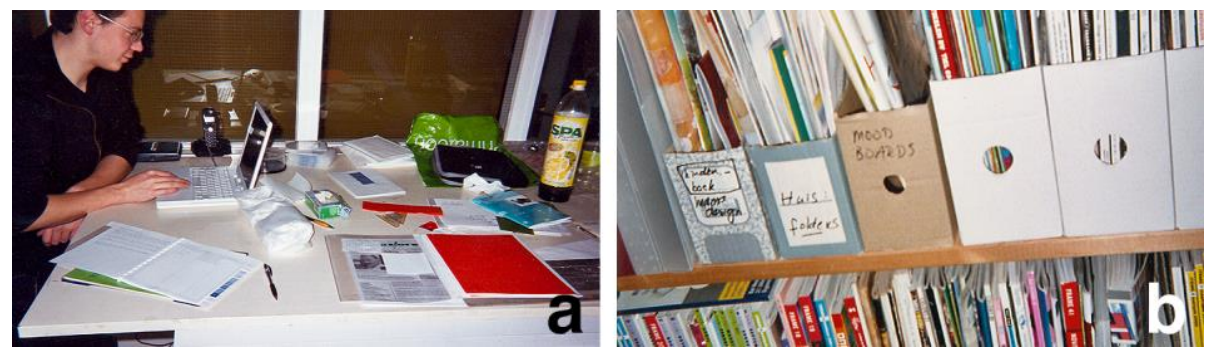

Fig. 1. a) Designing behind a standing desk. b) Bookshelves filled with magazines for inspiration, including a special box with magazines to make mood boards.

\subsection{Findings}

From the probes, a set of possible research directions connected to supporting creativity and finding inspiration in the early stages of the design process was deduced.

- Supporting flexibility in creation. Designers stressed the importance of working with their hands in the early stages of the design process. Designers prefer the naturalness of using pen and paper and thus keep a sketchbook at hand to make notes and bring their ideas to life. Work related to the creation phase is mostly performed away from the computer, as designers need tools that provide flexibility. This extends to their workplace, where designers want to decide how and where they work (Fig. 1a), and prefer not being tied to their computers or (indoor) physical spaces.

- Finding inspiration. This is important for designers. It is a way to forget about work for a while, allowing them to approach design problems from a different perspective with a fresh mind. Designers have different ways to find inspiration (e.g., browsing magazines and the web, reading books, visiting fairs, meeting people). Taking short breaks to perform physical activities both inside and outside the design studio can also be beneficial (e.g., playing darts or football at the office, taking a bicycle ride in town, walking the dog). Designers keep special areas in their studios where they can stick sketches, photos and project printouts, keep their collections of magazines, or relax for a while. Having a view on the outside world to observe people and life also creates an inspiring atmosphere inside the design studio.

- Mood boards. Designers mentioned the creation of mood boards as an important activity for their work. One designer described herself as a mood board designer, and kept special magazines to make her mood boards with (Fig. 1b). Mood boards (Fig. 2a, 2b) are an idea development tool consisting of visually stimulating images from magazines affixed to an A0 foam board, and which are used by designers and their clients to share their different views that emerge from the design brief [17].

The creation of mood boards was selected for further research, as it had not been sufficiently studied in design research despite its importance for designers. Furthermore, mood boards shared characteristics with the other findings: they are created in the early stages of the design process, by designers manipulating physical images, away from computers, and they force designers to move about their design studio. 

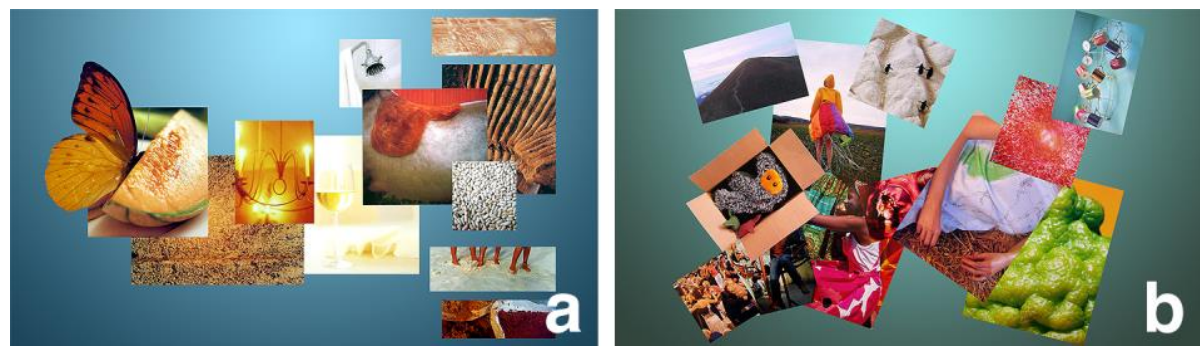

Fig. 2. Two mood board examples [17]: a) Exploring the use of other senses than just sight for interaction. b) The five roles that mood boards play at the start of the design process.

\section{Mood Boards}

After identifying the creation of mood boards as an important task for designers, an empirical study of how experienced designers from different design disciplines (i.e., fashion, textile and industrial design) use mood boards as part of their work was conducted. Earlier studies on mood boards have concentrated on understanding the role that mood boards play in design education [7], [19]. In doing so, these studies have been mostly looking at design students and how they create and perceive mood boards. One notable exception is Eckert and Stacey [6] who analyzed the use of mood boards to study the role played by sources of inspiration in the knitwear industry.

\subsection{Method, Participants and Procedure}

The type of study conducted was retrospective interviews based on contextual inquiry [10]. The retrospective interviews were conducted in the Netherlands and Finland with 14 practicing designers who regularly use mood boards as part of their work. All participants except one had at least ten years of design practice experience (14 years on average), with varying education level (university or academy), background (4 textile designers, 4 industrial designers, 3 fashion designers, 2 designers, and 1 stylist/photographer), age (between 35 and 45), and gender (9 female and 5 male). Three of them worked in large companies (i.e., Nokia and Stockmann), six of them worked in small design firms that they owned, and the rest did freelance work at home for large companies (e.g., Nike, SNCF, Rukka, Luhta, Pentik).

The retrospective interviews were planned for a total of two hours in the participants' workplace. Participants freely described between two and five of their previous projects for which they had used mood boards. Designers took the role of experts and guided the interviewer (the author) through different aspects of each mood board such as its purpose, the client's expectations, and the making process. There were no predefined sets of questions for the interviewer to ask, allowing for a more informal discussion to flow. Affinity diagramming [10] was used to analyze the data from the retrospective interviews. The interviewer plus two researchers first independently made notes as they watched the 14 interview videos, and then collaboratively analyzed the qualitative data through several interpretation rounds. 


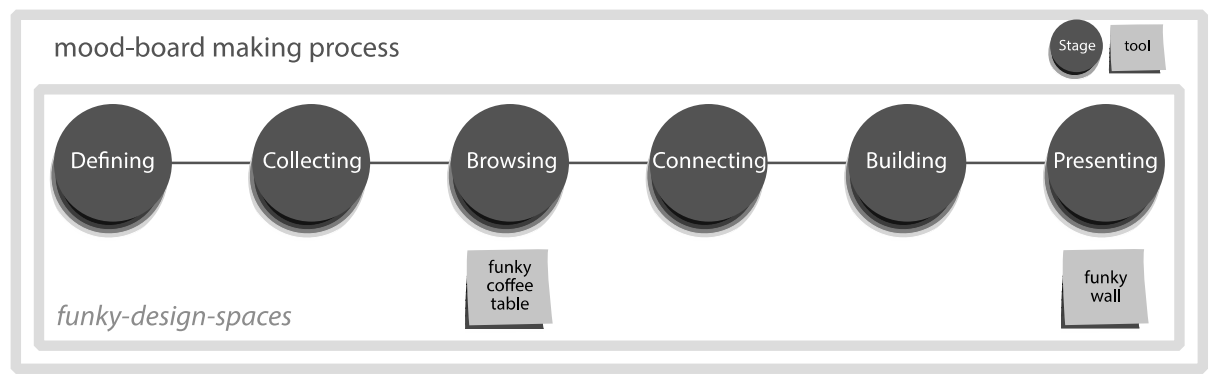

Fig. 3. The mood-board making process and the two support tools that were created. The Funky Coffee Table and the Funky Wall provide support for two stages of the process, i.e., browsing and presenting. The funky-design-spaces support the entire process creating mood boards.

\subsection{Findings}

Five Roles. Five roles that mood boards play in the early stages of the design process were identified [17]. First, mood boards play a framing role by defining the limits of the design task, which includes both problem setting and problem solving. Second, mood boards assist in the transmission of a mindset or vision by aligning the different stakeholders and getting them on the same wavelength. Third, mood boards support designers in visually researching apparently conflicting or contradicting ideas or paradoxing. Fourth, mood boards play an abstracting role by allowing designers to juxtapose both concrete and abstract imagery depending on the project and client. Finally, mood boards play a directing role by setting a trajectory for future design efforts. A mood board was created to communicate these five roles to different stakeholders (Fig. 2b) [17].

The Mood-Board Making Process. Six stages of the mood board making process were also found (Fig. 3). First, in defining, designer and client hold meetings where the client tries to express through words their rough ideas for a product or service, while the designer attempts to understand (and shape) what the client has in mind. Second, the designer begins by roughly collecting images that reflect the client's vision from magazines, the Internet, and occasionally their own personal collection of images (Fig. 4a). Third, once the designer feels they have enough (visual) material to work with, they will move on to browsing. Here the designer can spend a considerable amount of time pre-selecting images that will help them build a story or say something about the target audience, product, or company they are designing for. Fourth, through connecting, the designer sorts the pre-selected images in a simple and flexible way by assigning them to piles (usually up to 30 images per topic or idea). Fifth, the designer starts building the mood board by thinking how they want to arrange the images and create different layouts. In the final sixth stage, presenting, designer and client meet face-to-face to share and discuss the intended story behind the mood board (Fig. 4b). The designer creates a single large mood board or a series of smaller booklets for their clients to keep and share with stakeholders. 

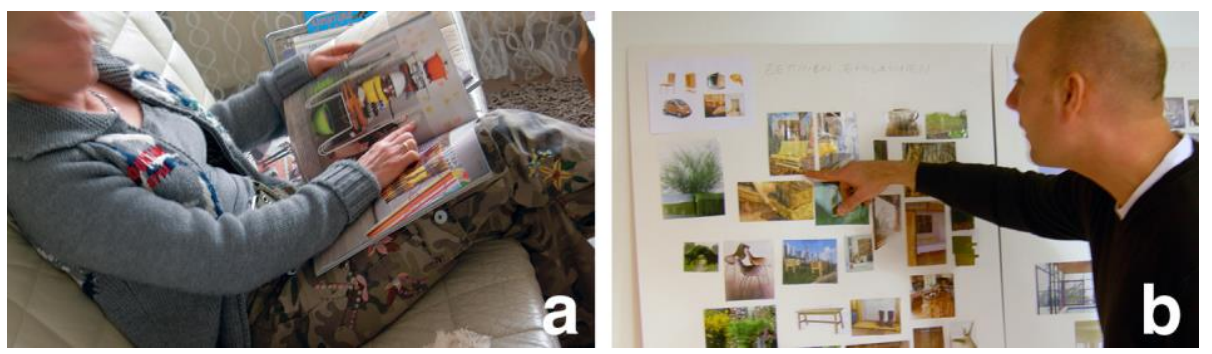

Fig. 4. a) A designer in her studio browsing images while seated on a couch by a coffee table. b) A designer guiding their client through the presentation by means of an explanation.

\subsection{Considerations for a Mood-Board Making Tool}

Based on the main aforementioned mood board interview findings, six considerations for a mood-board making tool were identified. Also, two mood boards were created to help communicate some of these findings to different stakeholders (Fig. 2a, 2b).

- Support Idea Development. Mood-board making entails a series of steps occurring both before and after building the mood board itself. New tools should support idea development through all six stages of the mood board making process, rather than only focus on the creation of the artifact.

- Encourage Two-Way Communication. Mood boards create the conditions for both designer and client to have a productive discussion together. New tools should en-courage two-way communication allowing the designer to present the mood board, see how the client perceives it, have a discussion, and get feedback on the artifact.

- Involving the Senses. Designers involve all five senses (plus their intuition) to create a mood board (e.g., they like the touch and smell of different paper types). However, mood-board creation on computers is currently restricted by the visual nature of the artifact. New tools should encourage using other senses than just sight for interaction.

- Holistic Interactive Space. Mood-board making is a dynamic and iterative process where designers must often switch activity and place. New tools should create a holistic interactive design space consisting of several interconnected tools that together support the mood-board making process.

- Merging With the Real Context. The process of making mood boards takes place at different locations inside and outside the design studio. New tools should merge with the real context of the activity by considering both place and the relaxed (e.g., image browsing on a couch) or formal (e.g., meeting or presentation) nature of the activity.

- Flexible and Intuitive Interaction. For creativity-related activities, designers prefer working with their hands using simple tools (e.g., pen and paper), and using their hands collaboratively (e.g., knife and fork). New tools should provide flexible and intuitive interaction through hand movements and other modalities (e.g., speech), allowing designers to perform tasks as naturally as they do now. 


\section{Co-Design}

Co-design activities with practicing designers and researchers were organized to collaboratively develop future ways of creating mood boards with novel technologies. Practitioners from different fields of research and design have understood the importance of involving diverse groups of users in the generation phase of novel artifacts, products and services, and thus facilitating participation has become one of the cornerstones of co-design [4]. Underpinning this approach is the supposition that stakeholders, including users, can contribute productively through involvement in the design process since they bring privileged insights into the domain that designers are trying to address and the ways in which future products and services may fit into and affect that domain. Within this field, there exists a variety of methods, techniques and events intended to inspire design participants and scaffold collaborative ideation and concept development. Such methods include inspiration card workshops [9], design games [3], and contextmapping [22].

\subsection{Method, Participants and Procedure}

The method used was dialogue-labs [16]. Dialogue-labs and its three structuring elements (i.e., process, space and materials), provide a structured way of generating ideas through a sequence of co-design activities. The process provides a clear step-bystep procedure for a two-hour idea-generation session in which participants work in pairs. The space is carefully crafted to align content to different locations, inspire participants and encourage them to move around the room. Finally, the materials are the means for participants to build a design language of their own and to provide different entry points to the design problem.

Seven dialogue-labs sessions were conducted in the Netherlands and Finland with 14 practicing designers, experienced mood-board makers, some of which had previously participated in the probes study $(n=1)$ and the retrospective interviews $(n=3)$. All participants had at least five years of design practice experience (ten years on average), with varying education level (university or academy), age (between 28 and 46) and gender (7 male, 7 female).

Each dialogue-labs session was planned for a total of two hours and involved four people; two designers plus two researchers who acted in a double role of facilitator/designer. Based on the findings from the previous studies with practicing designers (i.e., probes and retrospective interviews), activities and locations of the dialoguelabs environment were aligned according to the six stages of the mood-board making process (Fig. 3). The physical space was further designed to look and feel like a design studio (including working tables, chairs, a laptop, magazines, drawing materials, a large screen for presentation, and a sofa). Participants formed pairs, with at least one domain expert in them, and were then asked to think of unusual ways to support the process of making mood boards with novel technologies. At the end of each session, the quality of the resulting ideas was collectively assessed. Participants rated each idea on a seven-point Likert scale (where -3 was very bad, +3 was very good, and 0 was neutral). The most promising ideas (i.e., mean rating $\geq 2.5$ ) are presented below. 


\subsection{Ideas}

Layered Table. This tool consists of a set of adjustable multi-purpose surfaces (e.g., table, wall). Different surfaces can be used to keep a collection of materials, to make a selection of materials, to put the discarded material (e.g., garbage surface), or to build the mood board. Materials can easily be shared between surfaces. These surfaces stimulate designers to be standing in an active attitude to be able to easily and rapidly share materials between surfaces. Mood boards can be built on a horizontal surface, be put up on a vertical surface to check and share the results, and back down on the horizontal surface for adjusting. Surfaces with discarded material can inspire and be a starting point for other designers working on a different project.

Mood Sketching. This tool (Fig. 5a) supports the designer's creative impulses by allowing them to create several mood boards in a very quick and inexpensive way. Designers use a phone or tablet to intuitively sketch different moods by selecting, moving, rotating, and enlarging images on the screen. The sketched mood board is then shared and put up on a digital wall so that colleagues can comment on them. At this point, the mood board looks pixelated when enlarged for display, but it is only a sketch. When designers are happy with a few mood sketches, the materials used in them pop up on a large horizontal table in full resolution for fine-tuning and to create a final mood board.

Living Mood Board. The living mood board is a tool that makes mood boards become interactive living objects that provide inspiration and surprise. The designer creates a core mood board with a few basic elements, and then adds alternative images, movies or sounds for each basic element. The mood board is displayed on a wall and changes over time by reacting to context (e.g., the number of people around, the amount of light in the room, or if it has remained unchanged for some time). The tool and other colleagues can also suggest new content related to the basic elements to challenge the designer. The living mood board provides inspiration for its creator and other designers by catching their attention and defining the atmosphere they work in.

Presentation Recorder. This tool (Fig. 5b) helps client and designer interactively communicate and later remember ongoing discussions. The tool captures the presentation given by the designer and shares it with the client to also involve them in the process. It becomes some kind of contract with the agreement to follow a given path for the remainder of the project. This pre-editing tool only captures movements and sounds to see the discussion (i.e., no video, no faces). The client can then take specific things of the presentation, point at them and change them. The tool takes snapshots of the different stages of the discussion. Mood-board making becomes a closer cooperation between the designer and the client who work as a team. 


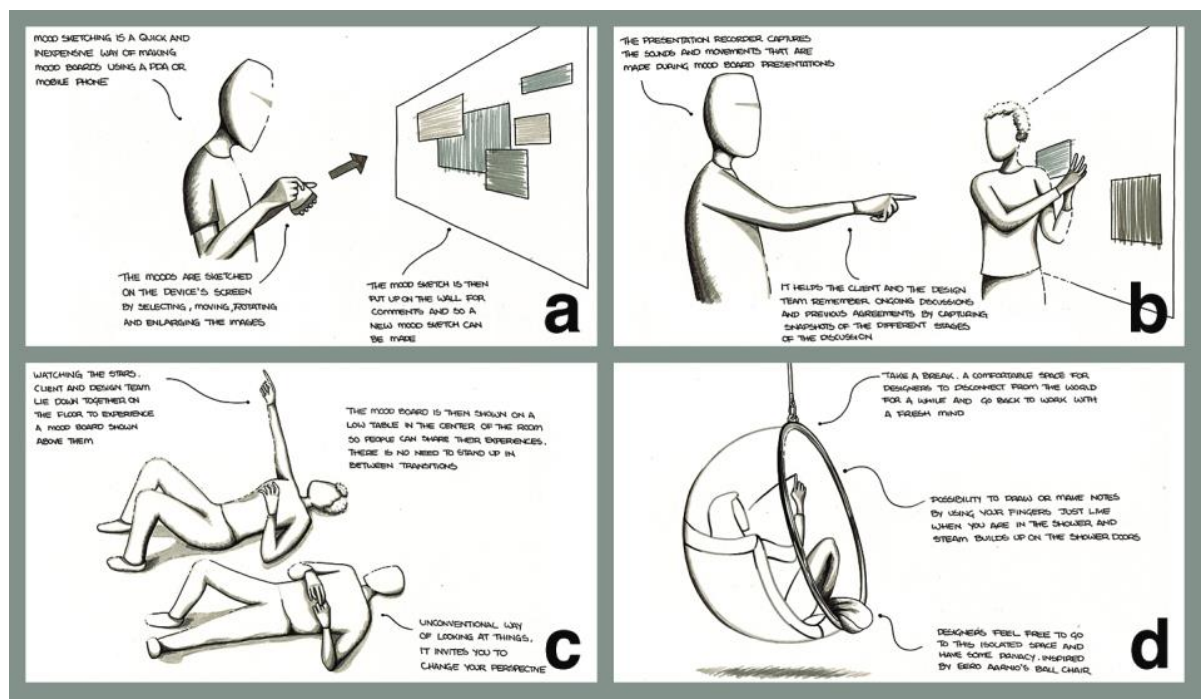

Fig. 5. The main ideas from the co-design sessions: a) In Mood Sketching designers use mobile devices to impulsively create several mood boards and put them up on the wall for feedback. b) Presentation Recorder captures sounds and movements made during a presentation, serving as a reminder of previous agreements. c) Watching the Stars is an invitation for designers and their clients to experience mood board presentations in a different way. d) Take a Break responds to the designers' need for a comfortable place where they feel free to rest and unwind for a while.

Watching the Stars. This tool (Fig. 5c) is an open invitation to experience mood boards in a new way. Client and designer let go of their inhibitions and lie down together on the floor (or on Japanese Tatami mats) in a completely different sensorial experience. The tool projects the mood board on the ceiling for a few minutes, allowing people to immerse themselves into the mood board as they observe in silence. Lying on the floor creates a more relaxed and intimate setting, almost like a personal cocoon. After witnessing the mood board, people sit around a low round table in the center of the room on which the mood board is projected while it slowly rotates to provide different views. After discussing and sharing their experiences of what they have seen, the mood board can be put up on the ceiling or down on the table once again for further discussion.

Take a Break. This tool (Fig. 5d) responds to the designers' need to have a space where they can momentarily disconnect from work, do something in there, and come back with a fresh mind. This comfortable space creates a feeling of disconnection from the world, similar to that of taking a shower or lying flat in bed. Designers feel free to go to this isolated space for a creative moment of doing nothing, while having some privacy and not being disturbed by others. An inspiration for this space could be Eero Aarnio's Ball Chair (1966), described as a "room within a room with a cozy and calm atmosphere, protecting outside noises and giving a private space for relaxing." 


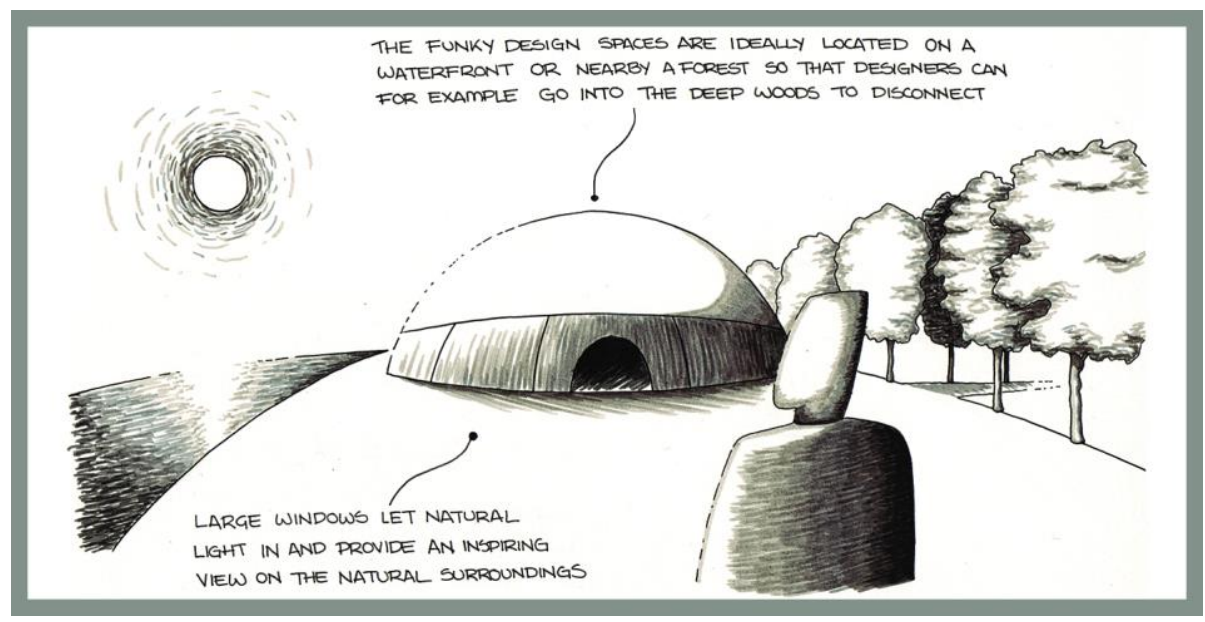

Fig. 6. The funky-design-spaces are located in a natural surrounding where designers can disconnect from the world by, for example, going into the deep woods. Large windows provide a direct view on the natural surroundings and allow natural light to energize designers.

\subsection{Funky-design-spaces}

The funky-design-spaces is a vision for a new holistic design studio, a comfortable environment that facilitates creative thinking in designers. During the co-design sessions, designers expressed the need to have easily convertible flexible spaces that support different mood-board making activities. The funky-design-spaces consist of interconnected tools that encourage breaking the rhythm [12] and stimulate designers to perform activities away from their desks. The co-design teams suggested a mentality change for their work culture where they would have the mental freedom to go outside during work hours to find inspiration or simply to take a break.

The funky-design-spaces are set in natural surroundings (Fig. 6) where designers can disconnect from the world and come back with a fresh mind. For example, designers can go into the deep woods or walk along the canal during work hours to reenergize. The dome-like shape of the environment is an open invitation to leave behind current conceptions of what a design studio is and think of new inspiring buildings that house the funky-design-spaces. Within this larger context, a houseboat on the canal or a greenhouse in the forest could become good examples of design studios that house the funky-design-spaces. Large windows provide a direct view on the natural surroundings and allow natural light to energize designers.

Once inside (Fig. 7), the funky-design-spaces create a relaxed and comfortable atmosphere where designers can engage in individual activities as well as creative collaboration. Designers feel that they belong to a larger team but they can also have a moment for themselves when needed. Adjustable multi-purpose surfaces can be used to easily display and share information. These surfaces can be assigned for different uses. 


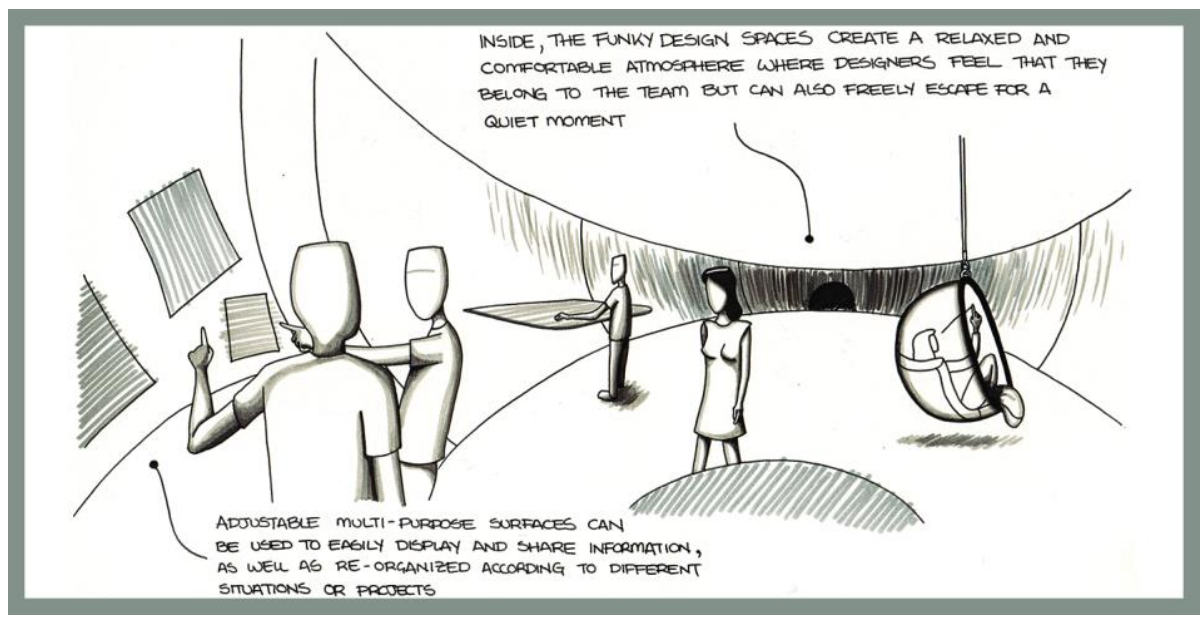

Fig. 7. The funky-design-spaces create a relaxed and comfortable atmosphere where designers can engage in individual activities as well as creative collaboration. Adjustable multi-purpose surfaces are used to easily display and share information.

Storing and moving information between these different tools or spaces should also be made in a simple way to avoid breaking the creative process (Fig. 8). Designers have a ball that they can bounce on a surface (e.g. wall) to collect information that is being displayed there. Then, designers can move to any other surface (e.g. table) and then roll the ball on the surface to display the contained information (e.g. images, audio, text, etc.).

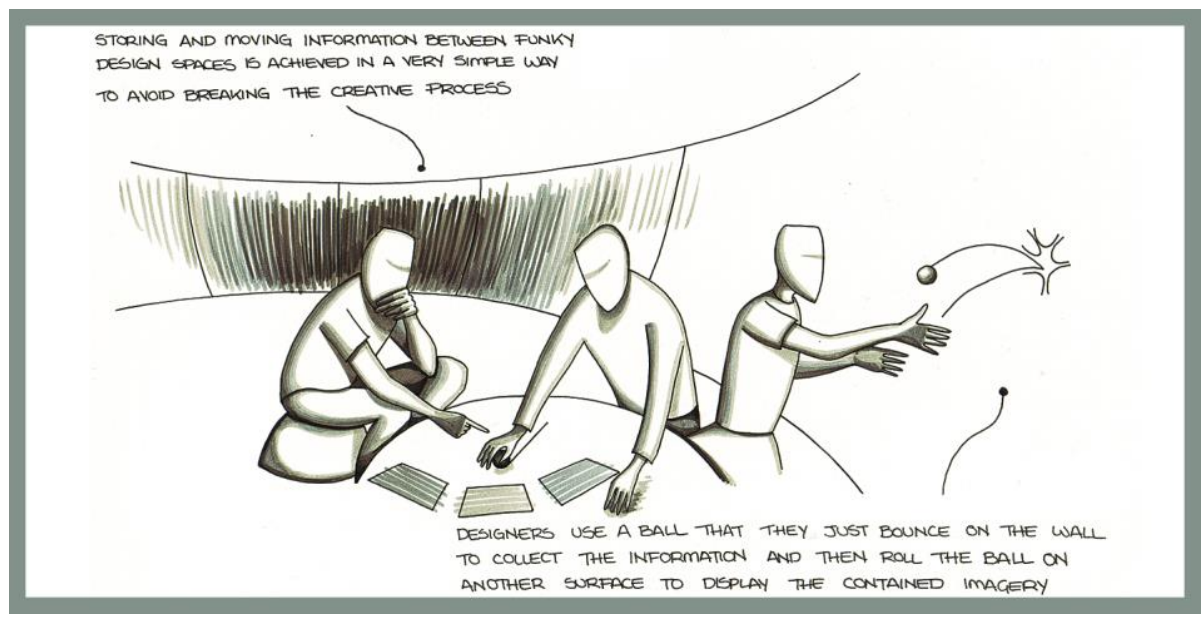

Fig. 8. Sharing information between the different funky-design-spaces is done in a simple way to support the natural creative flow. Designers have a ball that they can bounce on a wall to collect the information and then can move to a table and roll the ball on it to display the contained imagery. 


\section{$5 \quad$ Interactive Support Tools}

To test the ideas behind the funky-design-spaces, two prototypes (i.e., the Funky Coffee Table [14] and Funky Wall [15]) were developed and evaluated with designers.

\subsection{Intuitive Interaction}

Intuitive interaction is proposed as a perspective on providing interactive support for professional users in their work, and it consist of four parts. First, it allows people to simply walk up to and start interacting with a tool using their current skills and knowledge on the task that is being supported. There are no hidden functions, menus or complex actions to be learned, and thus engaging with the system just makes sense to them. Second, it allows designers to use their hands as the main input mechanism for activities that involve creation, through asymmetric two-handed interaction. Designers wear custom-made Lycra ${ }^{\circledR}$ gloves that contain sensors to detect hand gestures. Speech provides an alternative to interact with the tools. Third, it takes use contexts into account or the ability of the tools to merge with the existing possibilities of a design studio environment. Finally, the orthogonal distance from a surface (or ' $z$ ') is introduced as a cue for interaction, to generate extra interaction space, or to hide and reveal different functions of the tools.

\subsection{The Funky Coffee Table}

The Funky Coffee Table [14] is an interactive tabletop tool that supports image browsing (Fig. 3, 4a). A number of tabletop systems have been designed to support image browsing and sharing. The Personal Digital Historian [21] is a tabletop penbased system that helps people construct, organize, navigate and share digital collections in an interactive multi-person conversational setting. SharePic [1] is a multitouch, gestural, and collaborative digital photograph sharing application for a tabletop, which was strongly influenced by the way physical photographs are handled and placed on physical tables. Cabinet [12] helps designers collect and organize visual material for inspiration. Other authors [28], [20] have studied the general application of hand gestures and movements to support human-computer interaction.

Design. Three main principles guided its design. First, the interaction was set around a coffee table to encourage image searching in a relaxed setting (Fig. 9a). A long rectangular IKEA coffee table $(120 \times 40 \times 40 \mathrm{~cm}$.) was used, onto which three images were simultaneously projected to allow designers to discover, compare, and make connections between the materials. Second, the tool encourages designers to work using hand movements to pre-select images. Finally, to reduce the desk clutter that results from cutting out dozens of pictures from magazines (Fig. 4a), the space above the work surface has been extended for interaction and divided into multiple interaction layers [24]. Piles of images can be created in two layers above the table, which can be promptly consulted for an updated overview of the selection process. 


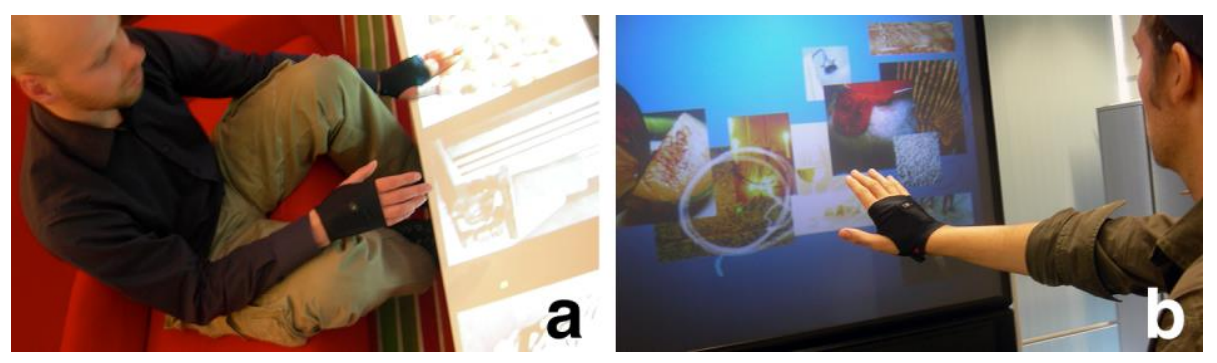

Fig. 9. a) In the Funky Coffee Table, designers use their hands to store images in layers above the table. b) Funky Wall uses hand gestures, body position, and speech to support presentations.

Interaction Techniques. There are two ways to browse images on the Funky Coffee Table. Designers can flip to the next or previous three images in the collection by placing their hand at table level and performing a short and quick diagonal movement to the left or to the right. The change of pages is accompanied by a page-flipping sound. Designers can also flick the collection by performing a longer and slower flip gesture, which triggers continuous image scrolling (without friction). The direction and speed of flicking are mapped to the direction and rate at which the images scroll. Continuous scrolling without friction was chosen to avoid fatigue while browsing large image collections. Tapping on the table stops continuous scrolling.

The Funky Coffee Table also allows creating, reviewing and arranging two soft piles of images using layers above the digital table. To put an image in a soft pile, the designer must place their dominant hand over an image at table-level and quickly move it upwards orthogonally with respect to the table surface. Depending on the highest point reached by the hand while performing the gesture, the image is placed into soft-pile A $(30-50 \mathrm{~cm})$ or B $(50-70 \mathrm{~cm})$. To review soft-pile contents, the designer must place their non-dominant hand above the table inside the range for layer A or B. Finally, to arrange soft piles, the designer must first use their non-dominant hand to locate the image and put their dominant hand on top of the image at the same orthogonal height as the non-dominant hand to activate it. By moving the dominant hand, the selected image can then be put into another soft-pile or removed from the pile.

\subsection{The Funky Wall}

The Funky Wall [15] is a wall-mounted display tool that supports presenting mood boards (Fig. 3, 4b). Clark and Brennan [5] have extensively studied the relation between gestures and speech, and the role of gestures in human communication. Von Hardenberg and Bérard studied bare-hand human computer interaction, focusing on static hand postures to issue a command, and fingers for pointing [26]. Vogel and Balakrishnan [25] studied shared interactive public displays that support the transition from implicit to explicit interaction. Hand gestures and touch were used for explicit interaction, while body orientation and location played part in implicit interaction. Charade [2] allows presenters to use free-hand gestures to control a remote computer display, while also using gestures to communicate with the audience. 
Design. Three main principles guided the design of the Funky Wall. First, four ranges of interaction are used depending on the designer's proximity to the wall display [25]: showing, contemplating, replaying, and exploring. Different tool functionalities are available to people (e.g., designer or client) in each range. Second, the designer records their presentation by gesturing and explaining in front of the screen, using their hands to point or outline specific areas of the mood board. The tool automatically records three essential aspects of the mood board presentation (i.e., gestures, speech, and visuals, which are used to split the presentation into a number of meaningful segments by looking into hand location, gesture acceleration, and speech pauses. Third, the tool supports two-way communication between designer and client by allowing them both to provide input by creating a presentation, and to share their thoughts by providing feedback. For this type of communication to happen, two Funky Walls are needed, one for the designer and another one for the client.

Interaction Techniques. In the first range of interaction (i.e., showing), the designer records a presentation by standing in front of the wall display at close range $(<0.5 \mathrm{~m})$ and then simply gesturing and speaking next to the mood board. The tool overlays white traces of the hand gestures made by the designer, at $30 \%$ opacity to allow good visibility of the mood board. Traces gracefully degrade to $25 \%$ opacity after $10 \mathrm{sec}-$ onds to make recent traces more prominent than older ones.

The next three ranges of interaction provide different ways to review the presentation contents. In contemplating, spectators (i.e., the designer or client) assess the mood board by standing away from the wall display $(>2 \mathrm{~m})$, for a more comfortable and clean overview. In replaying, spectators play back the entire presentation by approaching the wall display $(1.5-2 \mathrm{~m})$. Raising the dominant hand results in displaying a static representation of all gestures made during the presentation, semitransparent on top of the mood board. Raising the non-dominant hand triggers the complete recorded speech. By putting both hands together, the recorded speech is played and the transparent dynamic gestures unfold as the presentation progresses. In exploring, spectators browse specific parts of the presentation by taking one step closer towards the wall display $(0.5-1.5 \mathrm{~m})$. Pointing with the dominant hand to a given area in the mood board shows a static representation of the traces made in that area (Fig. 9b). These overlaid traces of gestures serve as guides for retrieval. The tool highlights both the explanations made by the designer just before (i.e., in white) and immediately after (i.e., in black) the currently selected gesture. Putting both hands together triggers the dynamic gestures together with its corresponding spoken explanation.

Implementation. Both prototypes were set up using a desktop PC, connected to a top-down projector displaying a 120x40 $\mathrm{cm}(1272 \times 424$ pixels $)$ image on a white IKEA table $(120 \times 40 \times 40 \mathrm{~cm})$ for the Funky Coffee Table, and to a back-projection screen of $200 \times 150 \mathrm{~cm}(1024 \times 768$ pixels $)$ for the Funky Wall. Both PCs also controlled an InterSense IS-600 ultrasonic tracking system to detect hands. Participants wore custom-designed Lycra ${ }^{\circledR}$ gloves that contained the sensors (Fig. 9a, 9b). Both applications were written in $\mathrm{C \#}$ and used OpenGL for visualization purposes. 


\subsection{Method, Participants, Procedure}

Prototype evaluations of both tools were conducted in the Netherlands with nine practicing designers, some of which had previously participated in the probes study $(\mathrm{n}=1)$, the retrospective interviews $(n=2)$, and the co-design workshops $(n=3)$. The participants had at least five years of design practice experience (13 years on average), with varying education level (university or academy), age (between 31 and 46), gender (7 male, 2 female), and handedness ( 7 right, 2 left). The evaluations were conducted individually and lasted on average one hour.

In the first part of the evaluation participants experienced both tools in counterbalanced order (i.e., five began with the table and four with the wall). Participants were asked to perform simple tasks such as change pages, start and stop scrolling, create piles and rearrange piles using 30 images for the Funky Coffee Table, and make a new mood board presentation, and explore an existing one for the Funky Wall (20 min. per tool). In the second part of the evaluation, participants were asked to share their views on the funky-design-spaces, specifically on the idea of supporting the process of making mood boards by having distributed interconnected tools (10 min.). Earlier studies where designers evaluated either tool are reported elsewhere [14,15].

\subsection{Findings}

Participants agreed with the vision of a holistic design studio housing interconnected tools that stimulate designers to break away from their desks. Designers also reflected on how these spaces could and should encourage collaboration with more people, not just designers: "The problem of [working] in front of a computer is that you are on your own. (...) Someone else could be sitting here (points) and another one there (points), and all could be browsing simultaneously. (...) You could use these (tools) for focus groups (...) so it's not just designers that can use it. It would also be a way to [bring] the world of the designer closer to the user." [P1] "You could use the table to browse images and magazines in less solitude. The same goes for the wall." [P6]

Regarding the use of the orthogonal distance from the interactive surface for interaction, participants identified both positive (i.e., extra space) and negative aspects (i.e., lack of physicality) of interacting in open space (i.e. midair). Regarding asymmetric two-handed interaction, designers found similarities between the tools and the use of one hand to select and the other hand to perform an action: "Although I want to have less chaos in my office, (...) at the same time I still want to see (how big) the pile of images is (...) and then move towards the screen where I make (the mood board). So for me it still needs to have the physical world that I have but you translated it into another physical world into using distance as a cue for interaction and I like that. Maybe I am missing the physicality of it (in midair). In a way the table is already a touch screen so it comes very close to what I want." [P4] "(Using hand gestures) feels good for me because it's a bit the same with (triggering sounds on the wall) and the table where you choose a layer with one hand and activate with the other." [P6]

The table and wall prototypes running alongside each other allowed participants to get a glimpse of and imagine how the tools might work together. Designers reflected 
on how information might be transferred from one tool to another: "I see this table in a meeting room for example (...) so you can make groups of images and then you put them on the wall and the (groups of) images appear there (...) on the wall. So it's like a selecting table." [P1] "If you have places within one room, it should be easily shareable and also somehow clear what the other is trying to communicate to you in any stage of the mood board making process. " [P3]

\section{Discussion}

\subsection{Supporting the Remaining Stages of the Mood-Board Making Process}

The full funky-design-spaces vision was inspired by and provides support for the six stages of the mood-board making process (Fig. 3). However, the two prototypes developed only support two stages of the process (i.e., browsing and presenting). To obtain a full understanding of the impact of the proposed holistic environment, four tools that support the remaining stages of the mood-board making process would need to be co-designed and evaluated (i.e., defining, collecting, connecting, and building). All six interconnected tools would need to be evaluated alongside each other to check whether people might naturally group back some of these tools together, so that instead of six tools, designers might only require four. Participants made some comments during the evaluations of the tools that might lead in this direction.

\subsection{Virtual Space Above Versus Around the Table}

Setting the interaction above the table created a problem in terms of the mental model we were trying to introduce. When rearranging an image pile, the action space is set in mid-air (i.e., holding both hands above the table to access a layer and interact with an image), while the perception space is located at table-level (i.e., image projected on the table). Participants instinctively tried to grab the projected image at table-level instead of layer-level. One way to circumvent this problem is to use ' $y$ ' instead of ' $z$ ' as a cue for interaction. Each row of images corresponding to one magazine or pile would be browsed horizontally using ' $x$ ', and then designers could use ' $y$ ' to move to other groups of images or magazines. Alternatively, marking menus [13] could be used to assign different actions to the eight points of the compass. East (E) and West (W) would correspond to browsing backward and forward (as is currently), while the remaining six cardinal (i.e., North or N, South or S) and ordinal (i.e., NE, SE, SW, and NW) directions could be used to put images in piles. For novice users, pressing and waiting would display a discoverable menu as a reminder of the different options available. These eight directions fall within the capacity of short-term memory and therefore can be remembered and learned very quickly. The actions can be performed almost automatically with eyes closed so that the attention is always on the work. Another improvement for the Funky Coffee Table could be to retrieve the layer contents using one's fingers by indicating the number of the layer on the table or the direction where the layer is located by pointing to it. 


\subsection{Proximity-Based Interaction}

Using the distance from the vertical display in the Funky Wall allowed designers to easily reveal different parts of the tool. However, participants mentioned some difficulties in knowing which range of interaction they were in (i.e., showing, contemplating, replaying, exploring) solely based on visual aspects, due to a lack of other feedback. This happened especially when designers would walk closer or further away from the tool without performing any hand gestures (i.e., keeping their hands in a resting position next to their body). One way to tackle this issue could be to add an extra sensor that would only track the designer's position with respect to the screen independent from hand gesturing. Another alternative would be to use other motion sensing devices (e.g., Kinect), which might allow us to design a different set of gestures to select and trigger different parts of the presentation by doing quick movements in mid-air pointing towards as specific sound or part of the presentation. Audio transitions could also indicate moving from one range of interaction to another.

\section{Conclusions}

This paper presents an example of surface computing support using a co-design approach by systematically involving end users throughout the design process. In a series of activities with fifty designers (i.e., probes study, retrospective interviews, codesign workshops, and prototype evaluations) the funky-design-spaces vision of a holistic design studio housing interconnected tools for creativity was arrived at by observing designers making mood boards. Evaluations suggest these environments could improve creativity and encourage collaboration, however more tools are needed to fully test the vision. Future research includes developing tools for all stages of the mood-board making process, and evaluating them in a design studio for a longer time.

\section{References}

1. Apted, T., Kay, J., Quigley, A.: Tabletop sharing of digital photographs for the elderly. In: SIGCHI conference on Human Factors in computing systems, pp. 781-790. ACM (2006)

2. Baudel, T., Beaudouin-Lafon, M.: Charade: remote control of objects using free-hand gestures. Communications of the ACM, 36(7), 28-35 (1993)

3. Brandt, E., Messeter, J.: Facilitating collaboration through design games. In: 8th conference on Participatory design, pp. 121-131. ACM (2004)

4. Brandt, E., Johansson, M., Messeter, J.: The design lab: re-thinking what to design and how to design. In: T. Binder, M. Hellström. Design spaces. Helsinki: Edita, 34-43 (2005)

5. Clark, H.H., Brennan, S.E.: Grounding in communication. Perspectives on socially shared cognition, 13(1991), 127-149 (1991)

6. Eckert, C., Stacey, M.: Sources of inspiration: a language of design. Design Studies 21, 5, 523-538 (2000)

7. Garner, S., McDonagh, D.: Problem Interpretation and Resolution via Visual Stimuli: The Use of 'Mood Boards' in Design Education. Art and Des. Education 20, 1, 57-64 (2001)

8. Gaver, B., Dunne, T., Pacenti, E.: Design: cultural probes. interactions, 6(1), 21-29 (1999) 
9. Halskov, K., Dalsgård, P.: Inspiration card workshops. In: 6th conference on Designing Interactive systems, pp. 2-11. ACM (2006)

10. Holtzblatt, K., Wendell, J., Wood, S.: Rapid Contextual Design. Morgan Kaufmann (2005)

11. Jordà, S., Geiger, G., Alonso, M., Kaltenbrunner, M.: The reacTable: exploring the synergy between live music performance and tabletop tangible interfaces. In: 1st international conference on Tangible and embedded interaction, pp. 139-146. ACM (2007)

12. Keller, I., Sleeswijk Visser, F., van der Lugt, R., Stappers, P.J.: Collecting with Cabinet: or how designers organise visual material, researched through an experiential prototype. Design Studies, 30(1), 69-86 (2009)

13. Kurtenbach, G., Buxton, W.: The limits of expert performance using hierarchic marking menus. In: INTERACT'93 and CHI'93 conference on Human factors in computing systems, pp. 482-487. ACM (1993)

14. Lucero, A., Aliakseyeu, D., Martens, J.B.: Augmenting mood boards: flexible and intuitive interaction in the context of the design studio. In: International Workshop on Horizontal Interactive Human-Computer Systems, pp. 147-154. ACM (2007)

15. Lucero, A., Aliakseyeu, D., Overbeeke, K., Martens, J.B.: An interactive support tool to convey the intended message in asynchronous presentations. In: International Conference on Advances in Computer Entertainment Technology, pp. 11-18. ACM (2009)

16. Lucero, A., Vaajakallio, K., Dalsgaard, P.: The dialogue-labs method: process, space and materials as structuring elements to spark dialogue in co-design events. CoDesign $8,1,1$ $23(2011)$

17. Lucero, A.: Framing, aligning, paradoxing, abstracting, and directing: how design mood boards work. In: Designing Interactive Systems Conference, pp. 438-447. ACM (2012)

18. Mattelmäki, T.: Design probes. Aalto University (2006)

19. McDonagh, D., Denton, H.: Exploring the degree to which individual students share a common perception of specific mood boards: observations relating to teaching, learning and team-based design. Design Studies 26, 1, 35-53 (2005)

20. Quek, F., McNeill, D., Bryll, R., Duncan, S., Ma, X. F., Kirbas, C., McCullough, K.E., Ansari, R.: Multimodal human discourse: gesture and speech. ACM Transactions on Computer-Human Interaction (TOCHI), 9(3), 171-193 (2002)

21. Shen, C., Lesh, N. B., Vernier, F., Forlines, C., Frost, J.: Sharing and building digital group histories. In: Computer supported cooperative work, pp. 324-333. ACM (2002)

22. Sleeswijk Visser, F., Stappers, P.J., Van der Lugt, R., Sanders, E.B.: Contextmapping: experiences from practice. CoDesign, 1(2), 119-149 (2005)

23. Streitz, N.A., Geißler, J., Holmer, T., Konomi, S., Müller-Tomfelde, C., Reischl, W., Rexroth, P., Seitz, P., Steinmetz, R.: i-LAND: an interactive landscape for creativity and innovation. In: SIGCHI conference on Human factors in computing systems, pp. 120-127. ACM (1999)

24. Subramanian, S., Aliakseyeu, D., Lucero, A.: Multi-layer interaction for digital tables. In: 19th symposium on User interface software and technology, pp. 269-272. ACM (2006)

25. Vogel, D., Balakrishnan, R.: Interactive public ambient displays: transitioning from implicit to explicit, public to personal, interaction with multiple users. In annual ACM symposium on User interface software and technology, pp. 137-146. ACM (2004)

26. Von Hardenberg, C., Bérard, F.: Bare-hand human-computer interaction. In Proceedings of the 2001 workshop on Perceptive user interfaces, pp. 1-8. ACM (2001)

27. Wellner, P.: Interacting with paper on the DigitalDesk. Communications of the ACM, 36(7), 87-96 (1993)

28. Wexelblat, A.: An approach to natural gesture in virtual environments. ACM Transactions on Computer-Human Interaction (TOCHI), 2(3), 179-200 (1995) 